\title{
Intravenous fluid prescription practices among pediatric residents in Korea
}

Jiwon M. Lee, MD', Younghwa Jung, MD'1, Se Eun Lee, MD', Jun Ho Lee, MD, PhD², Kee Hyuck Kim, MD, PhD³, Ja Wook Koo, MD, PhD, Young Seo Park, MD, PhD ${ }^{5}$, Hae II Cheong, MD, PhD ${ }^{1,6,7}$, II-Soo Ha, MD, PhD ${ }^{1,7}$, Yong Choi, MD, PhD', Hee Gyung Kang, MD, PhD ${ }^{1,6}$

${ }^{1}$ Department of Pediatrics, Seoul National University Children's Hospital, Seoul, ${ }^{2}$ Department of Pediatrics, CHA Bundang Medical Center, CHA University, Seongnam, ${ }^{3}$ Department of Pediatrics, National Health Insurance Corporation Ilsan Hospital, Goyang, ${ }^{4}$ Department of Pediatrics, Inje Unversity Sanggye Paik Hospital, Inje Unversity College of Medicine, Seoul, ${ }^{5}$ Department of Pediatrics, Asan Medical Center, University of Ulsan College of Medicine, Seoul, ${ }^{6}$ Research Center for Rare Diseases, Seoul National University Hospital, Seoul, ${ }^{7}$ Kidney Research Institute, Medical Research Center, Seoul National University College of Medicine, Seoul, Korea

Purpose: Recent studies have established the association between hypotonic fluids administration and hospital-acquired hyponatremia in children. The present paper investigated the pattern of current practice in intravenous fluid prescription among Korean pediatric residents, to underscore the need for updated education.

Methods: A survey-based analysis was carried out. Pediatric residents at six university hospitals in Korea completed a survey consisting of four questions. Each question proposed a unique scenario in which the respondents had to prescribe either a hypotonic or an isotonic fluid for the patient.

Results: Ninety-one responses were collected and analyzed. In three of the four scenarios, a significant majority prescribed the hypotonic fluids (98.9\%, $85.7 \%$, and $69.2 \%$, respectively). Notably, $69.2 \%$ of the respondents selected the hypotonic fluids for postoperative management. Almost all (96.7\%) selected the isotonic fluids for hydration therapy.

Conclusion: In the given scenarios, the majority of Korean pediatric residents would prescribe a hypotonic fluid, except for initial hydration. The current state of pediatric fluid management, notably, heightens the risk of hospital-acquired hyponatremia. Updated clinical practice education on intravenous fluid prescription is urgently required.

Key words: Pediatrics, Intravenous, Infusions, Fluid therapy, Hyponatremia, Maintenance

\section{Introduction}

Fluid management is an essential component of pediatric practice, especially in intensive care and postoperative contexts. The traditional maintenance fluid in pediatrics is $0.2 \%$ saline in 5\% dextrose water (DW), as based on the fact that sodium concentration of 30 $\mathrm{mEq} / \mathrm{L}$ approximates the sodium composition of human breast milk and cow's milk ${ }^{1)}$. Concerns arose, however, that a hypotonic solution carries risks of water overload and subsequent hyponatremia, which can lead to fatal outcomes ${ }^{2)}$. Moritz and Ayus ${ }^{2)}$ introduced the idea of using $0.9 \%$ saline as the maintenance fluid, and several succeeding studies, based on the results of randomized trials, have supported this level as the safer choice $^{3-5)}$. Yet, universal agreement on fluid sodium concentrations in cases of pediatric maintenance intravenous administration remains elusive. The nineteenth and most recent edition of Nelson Textbook of Pediatrics ${ }^{6}$, the most commonly referred textbook, still states that "the usual choices for maintenance fluid therapy in children are 0.5 normal saline (NS) and 0.2 NS" (p. 243), unless the patient has volume depletion, as based on
Corresponding author: Hee Gyung Kang, MD, PhD Department of Pediatrics, Seoul National University Children's Hospital, 101 Daehak-ro, Jongno-gu, Seoul 110-744, Korea

Tel: +82-2-2072-0658

Fax: +82-2-2072-0274

E-mail: kanghg@snu.ac.kr

Received: 15 September, 2012

Revised: 5 October, 2012

Accepted: 24 October, 2012

Copyright (C) 2013 by The Korean Pediatric Society

This is an open-access article distributed under the terms of the Creative Commons Attribution NonCommercial License (http://creativecommons.org/ licenses/by-nc/3.0/) which permits unrestricted noncommercial use, distribution, and reproduction in any medium, provided the original work is properly cited. 
Holliday's method ${ }^{1)}$. The 10th edition of the Korean Textbook of Pediatrics ${ }^{7)}$ specifies that hypotonic fluids must be avoided in intensive care contexts, even where children weigh less than $10 \mathrm{~kg}$. Considering that these textbooks are the sources of the primary guidelines for pediatric care, residents might not be as aware as they should be of the most current fluid prescription issues.

In the present study, we performed a survey-based analysis of maintenance fluid prescription practices, targeting resident trainees in pediatrics to evaluate their knowledge and understanding of current fluid therapy issues and awareness regarding hyponatremia.

\section{Materials and methods}

A paper-based survey comprising four questions was distributed to residents in-training at six university hospitals in Korea: Seoul National University Hospital, Asan Medical Center, Dongguk University Ilsan Hospital, CHA Bundang Medical Center, Myongji Hospital, and Inje University Ilsan Paik Hospital. Each question introduced a clinical scenario in which the respondent was to choose the most appropriate fluid: 1) a six-month-old baby with acute bronchiolitis, 2) a five-year-old child with Henoch-Schöenlein purpura and abdominal pain; 3) a three-year-old who just received a surgery from acute appendicitis, and 4) a one-yearold infant with acute gastroenteritis and dehydration (Appendix). In each scenario, the condition of oral intake impairment was assumed. The fluid options were as follows:

1) $0.45 \% \mathrm{NaCl}$ in $5 \% \mathrm{DW}$ (NAK2)

2) $0.2-0.25 \% \mathrm{NaCl}$ in $\mathrm{DW}$ (SD 1:4, NAK3)

3) $0.9 \% \mathrm{NaCl}$ (NS)

4) $0.9 \% \mathrm{NaCl}$ in $5 \% \mathrm{DW}$ (5\% NS) (Appendix).

The survey was designed and structured by Y. Choi (Appendix). The respondents were informed that their identities were to remain anonymous. The survey data were cross-tabulated by question and fluid option. For the purposes of the analysis, the solutions were categorized as either hypotonic (NAK2, NAK3) or isotonic (NS, 5\% NS).

\section{Results}

A total of 91 responses were collected. The respondents were evenly distributed among freshman, sophomores, and junior and senior residents. Fig. 1 depicts the fluids selection for each of the presented scenarios. In the first scenario, a six-month-old patient with acute bronchiolitis, 89 of 91 respondents (97.8\%) selected $0.2 \%$ NS. In this respiratory disease context, excess antidiuretic hormone (ADH) can be produced ${ }^{8)}$. The textbooks generally recommend 0.2\% NS in 5\% DW for patients weighing under $10 \mathrm{~kg}$, but also state that such conditions might be more safely treated with a higher sodium concentration ${ }^{6,7)}$. In these situations, 100 $\mathrm{mL} / \mathrm{kg}$ is the guideline for calculation of the fluid amount, taking into account evaporative losses from the lungs as well'.

For the second scenario, the five-year-old Henoch-Schöenlein purpura patient with acute abdominal pain, hypotonic fluids were selected by $85.7 \%, 45.1 \%$ of those choosing $0.2 \%$ NS. In this clinical setting, as oral intake usually is restricted due to pain, maintenance fluid is supplied intravenously rather than orally. The sodium concentration should be higher than $0.45 \%$, due both to the patient's age ( 5 years $)^{5-7)}$, and the fact that pain also is a well-known stimulant of $\mathrm{ADH}^{2)}$. The amount of fluid can vary, though the textbooks recommend that patients with possible subtle volume depletion receive $20 \mathrm{~mL} / \mathrm{kg}$ of isotonic fluid over 1-2 hours and then be switched to D5+1/2 NS at a standard maintenance rate ${ }^{6,7)}$.

The third scenario involves the choice of fluid for the non peros period after an abdominal operation. Hypotonic fluids were still the preponderant choice (in 69.2\% of cases) for postoperative management, and 41 of 91 respondents to the present survey selected $0.2 \%$ NS. A randomized controlled study by Yung and Keeley $^{3)}$ found that postoperatively, children can show greater declines in plasma sodium concentration than adult patients, and thus need to be treated with isotonic salines. The textbooks also recommend that surgical patients should receive isotonic fluids at least 6 to 8 hours postoperatively ${ }^{6}$. A significant majority of respondents prescribed a hypotonic saline for postoperative management, which can increase the risk of iatrogenic hyponatremia.

In the fourth scenario, that which required selection of a fluid for initial hydration of an infant with dehydration, the majority of respondents (96.7\%) selected isotonic fluids. In fact, isotonic fluid is uniformly recommended by the textbooks, and in previous reports, as the initial fluid of choice for treatment of dehydration $^{3,6,7,9)}$. In a prospective randomized study, Neville et al. ${ }^{5)}$ determined that isotonic fluid is better than hypotonic saline for intravenous rehydration of children with gastroenteritis, which conclusion is supported by later studies ${ }^{4,9]}$.

\section{Discussion}

With a simple questionnaire, we assessed the maintenancefluid prescription choices of pediatric trainees. Whereas most of the respondents chose isotonic fluid for initial hydration, in most of the other situations, $0.2 \%$ NS was selected far more commonly than $0.45 \%$ or $0.9 \%$ NS. The traditional sodium content of maintenance fluid was $20-30 \mathrm{mEq} / \mathrm{L}$, equivalent to $0.2 \% \mathrm{NS}$. However, this practice had incurred hospital-acquired hyponatremia in many reported cases, resulting in morbidity and even mortality, and 
prompting calls for review of clinical practices in fluid therapy ${ }^{10-}$

${ }^{12)}$. The conventional hypotonic fluid prescription for children was established before recognition of the significant potential for stimulation of arginine-vasopressin (AVP) production in most hospitalized patients ${ }^{11)}$. Common conditions such as pain, stress, starvation, fever, and surgery, as well as lung or central nervous system disease, are now known to stimulate AVP production ${ }^{11,13)}$. Hypotonic fluid administration in the presence of elevated AVP levels results in a sharp fall in the serum sodium level, whereas isotonic fluids effectively prevent $\mathrm{it}^{2,9,11,14)}$. Several prospective studies, moreover, have supported the hypothesis that $0.9 \% \mathrm{NaCl}$ is an effective prophylaxis against hyponatremia ${ }^{3,4)}$.

The four clinical scenarios presented in the survey were all examples of the above-noted AVP-excess conditions ${ }^{9,14)}$. Ill children can have multiple nonosmotic triggers for AVP secretion ${ }^{11}$.

A
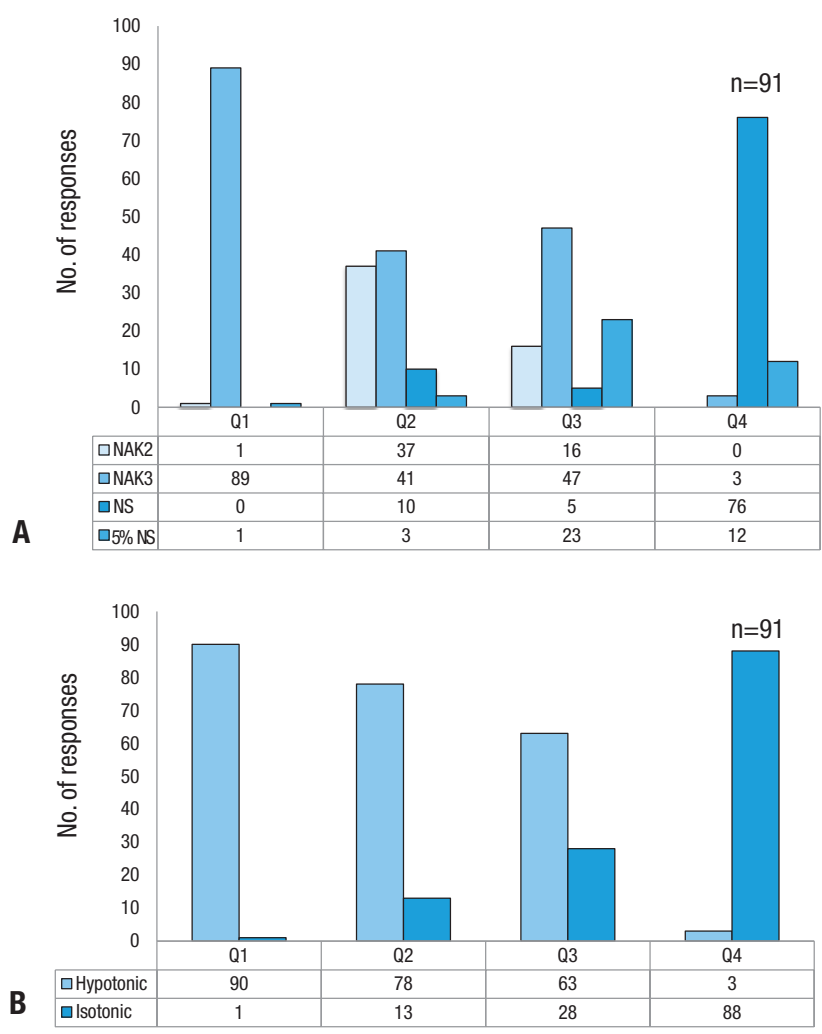

Fig. 1. Responses for each clinical scenario. The respondents predominantly selected hypotonic fluids in the first three cases. The responses are shown (A) by question and fluid option and (B) by fluid type: hypotonic or isotonic. NAK2 (1/2 NS in $5 \%$ dextrose water [DW]) contains dextrose $50 \mathrm{~g}$, Na 77 $\mathrm{mEq}, \mathrm{K} 20 \mathrm{mEq}$ in 1,000 mL of water; NAK3 (1/4 NS in 5\% DW) contains approximately dextrose $50 \mathrm{~g}$, Na $38 \mathrm{mEq}, \mathrm{K} 20 \mathrm{mEq}$ in 1,000 mL of water or SD 1:4 (1/5 NS in DW): contains approximately dextrose $40 \mathrm{~g}$, Na $30 \mathrm{mEq}$ in $1,000 \mathrm{~mL}$ of water; NS (0.9\% saline) contains Na $154 \mathrm{mEq}$ in 1,000 $\mathrm{mL}$ of water; $5 \%$ NS $(0.9 \%$ saline in $5 \%$ DW) contains dextrose $50 \mathrm{~g}, \mathrm{Na}$ $154 \mathrm{mEq}$ in 1,000 mL of water (NAK2, NAK3=hypotonic; NS=isotonic, glucose-free; $5 \%$ NS=isotonic with glucose). DW, NAK2 0.45\% NS in 5\% DW; NAK3/SD 1:4, 0.2-0.25\% NS in 5\% DW; NS, $0.9 \% \mathrm{NaCl} ; 5 \% \mathrm{NS}$, $0.9 \%$ NS in $5 \%$ DW (Appendix).
Among the 1,057 children admitted to the pediatric emergency room of Seoul National University Children's Hospital in 2006, excluding those with underlying diseases involving unstable electrolyte control such as chronic kidney disease, Bartters syndrome or other tubulopathy, 229 (21.7\%) showed hyponatremia (unpublished data). Moreover, whereas the brain develops to the adult size by the age of six, the skull does not catch up until adolescence ${ }^{15)}$. Such a high brain-to-intracranial-volume ratio increases the risk of encephalopathy, which is to say that children can develop encephalopathy with relatively milder hyponatremia ${ }^{8)}$. In this light, practitioners should be mindful that severe hyponatremia can occur in cases where hypotonic fluids are prescribed. Current pediatrics textbooks recommend higher than $0.2 \%$ sodium contents for patients who might be producing AVP owing to volume depletion or mechanisms such as stress, pain, nausea, or respiratory disease ${ }^{6,7)}$. However, the present survey results showed that a substantial proportion of Korean pediatric residents would routinely select hypotonic fluids in cases of common disease; furthermore, there were no considerable differences among the grades in training, which fact added weight to the necessity of up-to-date education. Freeman et al.'s ${ }^{16)} 2012$ survey of U.S. pediatric residents revealed a tendency to prescribe hypotonic fluids, though there was increasing awareness of the rationale for isotonic fluids. And in fact, residents who were aware of the fluid prescription controversy were twice as likely to select isotonic fluids as their colleagues who were not. To narrow the gap between academic debate and practical fluid management, programmed education and effective guidelines are necessary.

In many cases, "routine" maintenance fluid, simply, is chosen for every patient, without giving the matter much consideration. In a pediatric ward, the default fluid often is an NAK3 or SD 1:4, both of which are $0.2 \%$ NS. However, $0.2 \%$ NS can be too hypotonic; and indeed, with $0.2 \% \mathrm{NS}$, all four of the scenarios presented in our survey might show significant hyponatremia. Therefore, before intravenous fluid is prescribed, it needs to be considered whether such administration really is necessary for the patient; and, if necessary, the electrolyte concentration of the fluid, as well as the rate and amount of fluid administration, must be carefully determined. In fact, in many cases, intravenous fluid administration is not necessary provided that the patient has a sense of thirst and access to water. Notwithstanding, fluid therapy is the cornerstone of recuperation in all critically ill patients. It should be noted that isotonic fluid is less dangerous to most children than hypotonic fluid. In any event, it is critical to monitor a patient's electrolytes once intravenous fluid administration has been initiated. The overall findings of our survey suggest that current advances in fluid management have limited reach and application in the resident training environments and that they need to be emphasized and imparted, especially 
to front-line practitioners. We hope that the present work will alert clinicians and primary medical practitioners to the need for programmed education and updated clinical practice guidelines on intravenous fluid prescription.

\section{Conflict of interest}

No potential conflict of interest relevant to this article was reported.

\section{References}

1. Holliday MA, Segar WE. The maintenance need for water in parenteral fluid therapy. Pediatrics 1957;19:823-32.

2. Moritz ML, Ayus JC. Prevention of hospital-acquired hyponatremia: a case for using isotonic saline. Pediatrics 2003;111:227-30.

3. Yung M, Keeley S. Randomised controlled trial of intravenous maintenance fluids. J Paediatr Child Health 2009;45:9-14.

4. Kannan L, Lodha R, Vivekanandhan S, Bagga A, Kabra SK, Kabra M. Intravenous fluid regimen and hyponatraemia among children: a randomized controlled trial. Pediatr Nephrol 2010;25:2303-9.

5. Neville KA, Verge CF, Rosenberg AR, O'Meara MW, Walker JL. Isotonic is better than hypotonic saline for intravenous rehydration of children with gastroenteritis: a prospective randomised study. Arch Dis Child 2006;91:226-32.

6. Kliegman RM, Stanton B, St. Geme J, Schor N, Behrman RE. Nelson Textbook of Pediatrics. 19th ed. Philadelphia: Elsevier Saunders, 2011.

7. Hong CE. Textbook of pediatrics. 10th ed. Seoul: Korea Textbook Publishing Co., 2012.

8. Moritz ML, Ayus JC. The pathophysiology and treatment of hyponatraemic encephalopathy: an update. Nephrol Dial Transplant 2003;18:2486-91.

9. Moritz ML, Ayus JC. Improving intravenous fluid therapy in children with gastroenteritis. Pediatr Nephrol 2010;25:1383-4.

10. Moritz ML, Ayus JC. Hospital-acquired hyponatremia: why are there still deaths? Pediatrics 2004;113:1395-6.

11. Moritz ML, Ayus JC. Hospital-acquired hyponatremia: why are hypotonic parenteral fluids still being used? Nat Clin Pract Nephrol 2007;3:374-82.

12. Moritz ML. Reducing risks of hospital acquired hyponatremia. Pediatr Neurol 2005;33:75.

13. Moritz ML, Ayus JC. Disorders of water metabolism in children: hyponatremia and hypernatremia. Pediatr Rev 2002;23:371-80.

14. Choong K, Kho ME, Menon K, Bohn D. Hypotonic versus isotonic saline in hospitalised children: a systematic review. Arch Dis Child 2006;91:828-35.

15. Sgouros S, Goldin JH, Hockley AD, Wake MJ, Natarajan K. Intracranial volume change in childhood. J Neurosurg 1999;91:610-6.

16. Freeman MA, Ayus JC, Moritz ML. Maintenance intravenous fluid prescribing practices among paediatric residents. Acta Paediatr 2012;101:e465-8. 